\title{
Application of variational iteration method and electron transfer mediator/catalyst composites in modified electrodes
}

\author{
Alagu Eswari, Lakshmanan Rajendran*
}

The Madura College, Madurai, India; *Corresponding Author: raj_sms@rediffmail.com

Received 20 February 2010; revised 23 March 2010; accepted 23 April 2010.

\begin{abstract}
The nonlinear coupled system of diffusion equations are solved analytically for the transport and kinetics of electrons and reactant in the layer of a modified electrode. Analytical expressions of concentrations of substrate and mediator are presented using He's variational iteration method. The approximate expression of current for microheterogeneous catalysis at isonomer or redox polymer modified electrodes is also obtained. The results of the available limiting cases are compared with our results and are found to be in good agreement.
\end{abstract}

Keywords: Variational Methods; Nonlinear Boundary Value Problems; Simulation; Reaction-Diffusion Equations; Mathematical Modeling

\section{INTRODUCTION}

Recently the electrocatalytic activity of polymer modified electrodes has been the subject of considerable study of many researchers. Generally most systems are used to require an efficient electron transfer mediator in addition to displaying good electrocatalytic activity. We can find many features for electrocatalysis in the use of microscopic particles of metals or metal oxides dispersed within polymeric films deposited on electrode surfaces. This dispersion of catalytic materials offers important catalytic advantages. We are interested in the design of such microheterogeneous systems for efficient electrocatalysis.

A modified electrode differs from an ordinary electrode by having a thin film of some coated material which prevent direct contact between the metal surface and the bulk electrolyte. Electrochemical reactions of species in solution take place through that thin film. Instead of a direct electron transfer between the Fermi level of the metal and the ion in solution, the electron transfer is 'mediated' by the redox groups present in the thin layer. A large number of different modified electrodes have been made and certain systems have received more attention than others.

Lyons, McCormack, and Bartlett [1] presented an analytical model which quantified the transport and kinetics in conducting polymer modified electrodes containing a homogeneous distribution of spherical microparticulate catalysts. In their paper Lyons, McCormack, and Bartlett [1] obtained the analytical expressions of the substrate and mediator concentrations for the different values of parameters. Lyons and Bartlett [2] also presented the analytical expressions of substrate and mediator concentrations only for limiting values of dimensionless parameters. The transport and kinetics of reactions in chemically modified electrodes have been analyzed previously by Lyons and co-workers and approximate analytical solutions are available [3-7] .

In this paper we analyze application of catalyst composites in modified electrodes. To date many researches have been done on the application of modified electrodes. To my knowledge no rigorous analytical solutions for substrate and mediator concentrations have been reported in that application. Hence the main objective of this paper is to derive the analytical expressions of concentrations of substrate and mediator for all values of parameters using variational iteration method. The approximate expression of current for microheterogeneous catalysis at isonomer or redox polymer modified electrodes is also obtained for all values of dimensionless parameters $\beta$ and $\gamma$ (these parameters are defined in the (7)).

\section{MATHEMATICAL FORMULATION OF THE PROBLEM AND ANALYSIS}

Steady state boundary value problems described the tra- 
nsport and kinetics within the film can be written in dimensionless form as follows [2]:

$$
\begin{gathered}
D_{A} \frac{d^{2} a}{d x^{2}}-\frac{4 \pi r_{0}{ }^{2} N D_{A}\left(k_{A, 0}^{\prime} k_{S, 0}^{\prime}\right)^{1 / 2}(a s)^{1 / 2}}{\left[D_{A}+r_{0}\left(k_{A, 0}^{\prime} k_{S, 0}^{\prime}\right)^{1 / 2}(s / a)^{1 / 2}\right]}=0 \\
D_{S} \frac{d^{2} s}{d x^{2}}-\frac{4 \pi r_{0}{ }^{2} N D_{S}\left(k_{A, 0}^{\prime} k_{S, 0}^{\prime}\right)^{1 / 2}(a s)^{1 / 2}}{\left[D_{S}+r_{0}\left(k_{A, 0}^{\prime} k_{S, 0}^{\prime}\right)^{1 / 2}(a / s)^{1 / 2}\right]}=0
\end{gathered}
$$

where $k_{A}^{\prime}$ and $k_{S}^{\prime}$ are electrochemical rate constant, $r_{0}$ is the radius of an electrode, $D_{S}$ is the diffusion co-efficient for the substrate concentration, $D_{A}$ is the diffusion co-efficient for the mediator, $N$ is the number of particles per unit volume, $a$ is the concentration of the mediator and $s$ is the concentration of the substrate. These coupled non-linear differential equations have to be solved by applying the following boundary conditions:

$$
x=0, d s / d x=0 \text { and } a=a^{\infty}
$$

and

$$
x=L, \quad s=s^{\infty} \text { and } \mathrm{d} a / \mathrm{d} x=0
$$

The following dimensionless parameters for substrate concentration $u$, mediator concentration $v$, and distance $X$ are introduced:

$$
u=s / s^{\infty}, v=a / a^{\infty}, X=x / X_{K}
$$

where $X_{k}$ denotes a reaction layer thickness defined by the relation:

$$
X_{k}=\left(\frac{1}{4 \pi r_{0} N}\right)^{1 / 2}
$$

We also introduce the dimensionless parameters $\beta$ and $\gamma$ defined according to the following relations:

$$
\beta=\left(a^{\infty} D_{A} / s^{\infty} D_{S}\right)^{1 / 2}, \quad \gamma=r_{0}\left(k_{A, 0}^{\prime} k_{S, 0}^{\prime} / D_{A} D_{S}\right)^{1 / 2}
$$

Now the Eq.1 and Eq.2 reduce to the following dimensionless form:

$$
\frac{d^{2} u}{d x^{2}}-\frac{\gamma \beta(u v)^{1 / 2}}{1+\gamma \beta(v / u)^{1 / 2}}=0
$$

for the substrate, and

$$
\frac{d^{2} v}{d x^{2}}-\frac{\gamma(u v)^{1 / 2}}{\beta+\gamma(u / v)^{1 / 2}}=0
$$

for the mediator respectively. Here we can assume that the reaction layer thickness $X_{K}=L$. Now the boundary conditions may be expressed as follows:

$$
\begin{aligned}
& x=0, v=1 \text { and } d u / d x=0 \\
& x=1, d v / d x=0 \text { and } u=1
\end{aligned}
$$

The flux $j$ is given by

$$
j=-D_{A}(d a / d x)_{x=0}=D_{S}(d s / d x)_{x=L}
$$

or in non-dimensional form:

$$
\begin{aligned}
j & =-D_{A} a^{\infty}\left(4 \pi r_{0} N\right)^{1 / 2}(d v / d x)_{x=0} \\
& =D_{S} s^{\infty}\left(4 \pi r_{0} N\right)^{1 / 2}(d u / d x)_{x=1}
\end{aligned}
$$

The required expression of the normalized current is

$$
I=\frac{j}{D_{S} s^{\infty}\left(4 \pi r_{0} N\right)^{1 / 2}}=(d u / d x)_{x=1}
$$

or

$$
I=\frac{j}{D_{A} a^{\infty}\left(4 \pi r_{0} N\right)^{1 / 2}}=-(d v / d x)_{x=0}
$$

\subsection{Case by Case Transport and Kinetics Analysis}

\section{Case-1: Transport and Kinetics of the Substrate with- in the Layer}

We consider initially the master Eq.8 describing the transport and kinetics of the substrate in the layer when $\gamma \beta<<1$. Eq.8 can be written as

$$
\frac{d^{2} u}{d x^{2}}-(\gamma \beta) u^{1 / 2} v^{1 / 2}+\left(\gamma^{2} \beta^{2}\right) v=0
$$

Similarly when $\gamma \beta>>1$ (or) $1 / \gamma \beta<<1$, the (8) reduces to

$$
\frac{d^{2} u}{d x^{2}}-u+(1 / \gamma \beta) u^{1 / 2} v^{-1 / 2}=0
$$

The above equations are non-linear and only approximation solutions may be found. Using variational iteration method (Appendix-A), we obtain the concentration of the substrate (by solving the Eq.16)

$$
\begin{aligned}
u(x)= & (1+a)^{2}-p\left(0.033 a^{2} x^{6}-0.1 a^{2} x^{5}\right. \\
& -0.083 a x^{4}-0.083 a^{2} x^{4}+0.083 a x^{4} \\
& \left.+0.33 a x^{3}+0.33 a^{2} x^{3}-0.5 x^{2}-0.5 a x^{2}\right) \\
& +p^{2}\left(-0.033 a^{2} x^{6}+0.2 a^{2} x^{5}-0.167 a x^{4}\right. \\
& \left.-0.33 a^{2} x^{4}+0.667 a x^{3}-0.5 x^{2}\right)
\end{aligned}
$$

when $\gamma \beta=p<<1$. Using the boundary condition (11) we obtain the following relation between $p$ and $a$.

$$
\begin{aligned}
a= & -0.25 p+0.239 p^{2}+0.108 p^{3} \\
& -0.076 p^{4}-0.075 p^{5}
\end{aligned}
$$

From the above relation we obtain the values of $a$ for any given values of $p<<1$. The numerical values of $a$ for some given values of $p$ are given in Table 1. When $x$ is small, concentration of the substrate (when $\gamma \beta=p<<1$ ) Eq.18 becomes 


$$
u(x) \approx(1+a)^{2}+0.5 p(1+a-p) x^{2}
$$

Also from the above Eq.20 $u \approx 1$ when $\gamma \beta=p=0$ $(a=0)$ and $x=1$. When $\gamma \beta \rightarrow 0$, the Eq.8 or Eq.16 becomes $d^{2} u / d x^{2}=0$. The solution of this equation using the boundary conditions (10) and (11) becomes $u=1$. This result is exactly equal to our result when $\gamma \beta \rightarrow 0$. Similarly, the concentration of the substrate becomes (by solving the Eq.17

$$
\begin{aligned}
u(x)= & (1+a)^{2}+\left(0.5+0.5 a^{2}+a-0.5 r-0.5 a r\right) x^{2} \\
& -0.333 a r(1+a) x^{3}+\left(-0.1667 a^{2}-0.1667 a\right. \\
& \left.+0.1667 a r-0.25 a^{2} r-0.333 a^{3} r\right) x^{4}+\left(0.3 a^{2} r\right. \\
& \left.-0.2 a^{3} r-0.4 a^{4} r\right) x^{5}+0.033 a^{2} x^{6}
\end{aligned}
$$

when $\gamma \beta>>1$ (or) $1 / \gamma \beta=r<<1$. Using the boundary condition (11), we obtain the following relation between $r$ and $a$.

$$
\begin{aligned}
& 0.4 a^{4} r+0.533 a^{3} r-(1.3663-0.283 r) a^{2} \\
& -(2.8333-0.6663 r) a-0.5(1-r)=0
\end{aligned}
$$

From the above relation we obtain the values of $a$ for any given values of $r<<1$. We can find the numerical values of $a$ through some specific values of $r$ as shown in Table 1. When $x$ is small, Eq.21 becomes

$$
u(x) \approx(1+a)\left[1+a+0.5(1+a-r) x^{2}-0.333 a r x^{3}\right]
$$

Also from the above Eq.23, $u \approx 1$ when $1 / \gamma \beta=r=0$ ( $a=-1.878$ or -0.195 ) and $x=1$. When $\gamma \beta \rightarrow \infty$, the Eq.8 or Eq.17 becomes $d^{2} u / d x^{2}-u=0$. The solution of this equation using the boundary conditions (10) and (11) becomes $u=1$. This result is exactly equal to our result when $\gamma \beta \rightarrow \infty$. These approximants for the concentra- tion of the substrate Eq.20, Eq.23 are the simplest closed- form of analytical approximation for $\gamma \beta<<1$ and $\gamma \beta>1$.

Case 2: Transport and Kinetics of the Mediator Within the Layer

We consider the master (9) describing the transport and kinetics of the mediator with in the layer when $\gamma / \beta<<1$. Now the (9) takes the form

$$
\frac{d^{2} v}{d x^{2}}-(\gamma / \beta) u^{1 / 2} v^{1 / 2}+\left(\gamma^{2} / \beta^{2}\right) u=0
$$

where as when $\gamma / \beta>>1$ (9) becomes

$$
\frac{d^{2} v}{d x^{2}}-v+(\beta / \gamma) v^{3 / 2} u^{-1 / 2}=0
$$

Figure 1 shows our schematic representation of the differential equations describing the transport and kinetics in a microheterogeneous system. Each of these expressions represents the approximations to the set of master equations outlined in the (8) and (9). Concentration of the mediator using variational iteration method (Appendix -A) becomes (by solving the Eq.24

$$
\begin{aligned}
v(x)= & 1-4 a x-q\left(0.033 a^{2} x^{6}-0.1 a^{2} x^{5}\right. \\
& -0.083 a x^{6}-0.083 a^{2} x^{4}+0.083 a x^{4} \\
& \left.+0.33 a x^{3}+0.33 a^{2} x^{3}-0.5 x^{2}-0.5 a x^{2}\right) \\
& +q^{2}\left(-0.033 a^{2} x^{6}+0.167 a x^{4}+0.167 a^{2} x^{4}\right. \\
& \left.-0.5 x^{2}-a x^{2}-0.5 a^{2} x^{2}\right)
\end{aligned}
$$

when $\gamma / \beta=q<<1$. Using the boundary condition (11) we obtain the following relation between $a$ and $q$

$$
a=0.25 q-0.239 q^{2}-0.099 q^{3}+0.078 q^{4}
$$

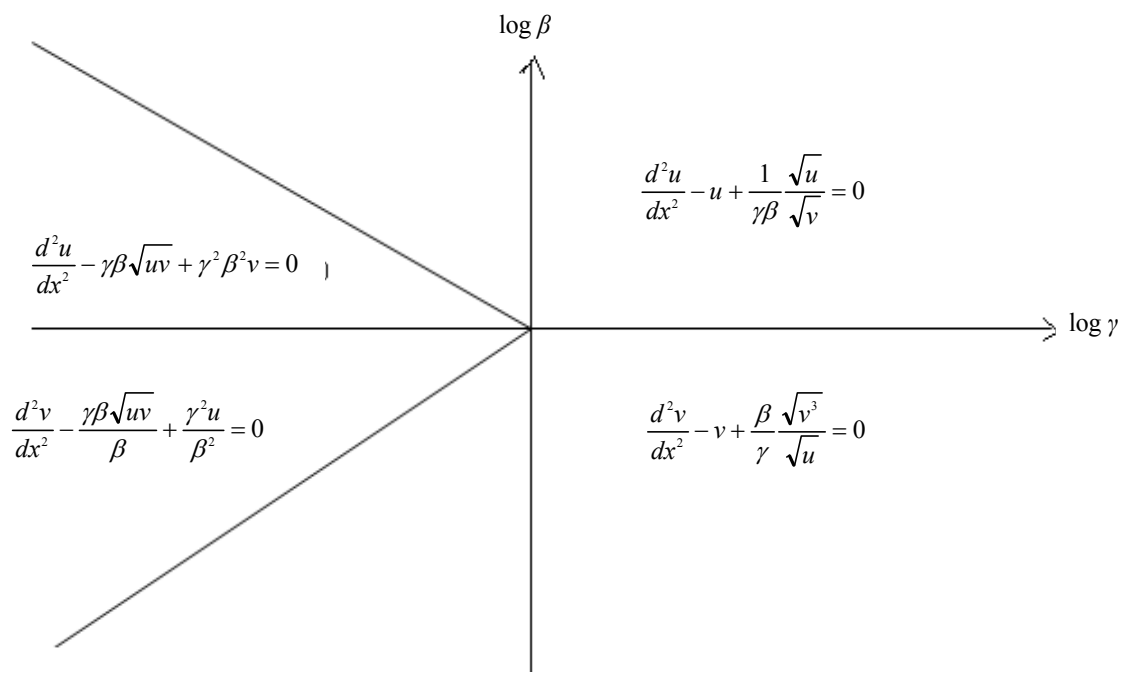

Figure 1. Our schematic representation of the differential equations describing the transport and kinetics in a microheterogeneous system. Each of these expressions represents approximations to the set of master equations outlined in the Eqs.8 and 9. 
The numerical values of $a$ for some given values of $q$ are given in Table 1. When $x$ is small, Eq.26 becomes

$$
v(x) \approx 1-4 a x+0.5 q\left(1+a-q-2 a q-a^{2} q\right) x^{2}
$$

Also from the above Eq.28, $v \approx 1$ when $\gamma / \beta=q=0$ $(a=0)$ and $x=0$. When $\gamma / \beta \rightarrow 0$, the Eq.9 or Eq.24 becomes $d^{2} v / d x^{2}=0$. The solution of this equation using the boundary conditions (10) and (11) becomes $v=1$. This result is exactly equal to our result when $\gamma / \beta \rightarrow 0$. Similarly, the concentration of the mediator becomes (by solving the Eq.25)

$$
\begin{aligned}
v(x)= & 1-4 a x+\left[0.5-0.5 l\left(1-a+a^{2}-a^{3}\right)\right] x^{2} \\
& +\left[-0.667 a+1.0002 a l\left(1-a+a^{2}-a^{3}\right)\right] x^{3} \\
& +\left[0.333 a^{2}+0.1667 a-0.083 l\left(4 a+7 a^{2}\right.\right. \\
& \left.\left.-6 a^{3}+9 a^{4}-12 a^{5}\right)\right] x^{4}+\left[-0.2 a^{2}-0.05 l\right. \\
& \left.\left(16 a^{3}-22 a^{4}+4 a^{5}+8 a^{6}-18 a^{2}\right)\right] x^{5} \\
& +0.033 a^{2} x^{6}
\end{aligned}
$$

when $\gamma / \beta>>1$ (or) $\beta / \gamma=l<<1$. Using the boundary condition (11) we obtain the following relation between $l$ and $a$

$$
\begin{aligned}
& 2 a^{6} l-2.996 a^{5} l+0.497 a^{4} l \\
& -1.998 a^{3} l+(1.831 l-0.533) a^{2} \\
& -(2.668 l-5.333) a-1+l=0
\end{aligned}
$$

We can find the numerical values of $a$ through some specific values of $l$ as shown in Table 1. When $x$ is small, Eq.29 becomes

$$
\begin{aligned}
& v(x) \approx 1-4 a x+0.5\left[1-l\left(1-a+a^{2}-a^{3}\right)\right] x^{2} \\
& +\left[-0.667 a+a l\left(1-a+a^{2}-a^{3}\right)\right] x^{3}
\end{aligned}
$$

Also from the above Eq.31 $v \approx 1$ when $\beta / \gamma=l=0$ ( $a=0.191$ or 9.814 ) and $x=0$. When $\beta / \gamma \rightarrow \infty$, the (9) or Eq.25 becomes $d^{2} v / d x^{2}-v=0$. The solution of this equation using the boundary conditions (10) and (11) becomes $v=1$. This result is exactly equal to our result when $\beta / \gamma \rightarrow \infty$. Eqs.28 and 31 represent the approximate new analytical expression of the concentration of the mediator when $\gamma / \beta=q<<1$ and $\beta / \gamma=l<<1$. Concentration of substrate and mediator are summarized in Table 2 and Table 3. Using Eqs.14 and 15 the nor-

\begin{tabular}{|c|c|c|c|c|c|c|}
\hline \multirow{2}{*}{ s.no } & \multirow{2}{*}{ Conditions } & \multicolumn{4}{|l|}{$u(x)$} & \multirow[t]{2}{*}{ Figures } \\
\hline & & This work & & Lyons and Bartlett [2] & & \\
\hline 1. & $\begin{array}{c}\gamma \beta<<1 \\
\quad \text { (or) } \\
\gamma \beta=p \leq 1\end{array}$ & $\begin{array}{l}\qquad u(x)=(1+a)^{2}+0.5 p(1+a-p) x^{2} \\
\text { where } \\
a=-0.25 p+0.239 p^{2}+0.108 p^{3}-0.076 p^{4}-0.075 p^{5}\end{array}$ & (20) & $u(x)=[1-0.2887 \sqrt{p}(1-x)]^{4}$ & (40) & $\begin{array}{c}\text { Figure } 3 \\
\gamma \beta=0.01,0.25,0.5 \\
\text { Figure } 11 \\
\gamma \beta=0.5\end{array}$ \\
\hline 2. & $\begin{array}{c}\gamma \beta>>1 \\
\quad(\text { or) } \\
1 / \gamma \beta=r \leq 1\end{array}$ & 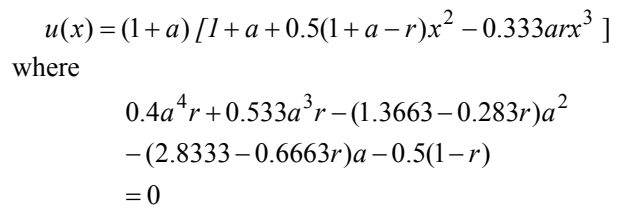 & (23) & $u(x)=\cosh (x) \sec h$ & (41) & $\begin{array}{l}\text { Figure } 4 \\
\gamma \beta=2,4,100 \\
\text { Figure } 12 \\
\gamma \beta=4\end{array}$ \\
\hline
\end{tabular}
malized current $I$ for various cases is given by

$$
\begin{aligned}
& I=\left(1-0.367 a^{2}\right) p+\left(-0.533 a^{2}+1.333 a-1\right) p^{2} \\
& \text { for } p=\gamma \beta<<1 \\
& I=4 a \quad \text { for } q=\gamma / \beta<<1 \\
& I=1-r+0.533 a^{2}+1.3332 a-1.3322 a r-0.499 a^{2} r \\
& -2.332 a^{3} r-2 a^{4} r \quad \text { for } r=1 / \gamma \beta<<1 \\
& I=4 a \quad \text { for } l=\beta / \gamma<<1
\end{aligned}
$$

The expression of the current is summarized in Table 4 and Table 5.

Table 1. Numerical values of $a$ for various values of $p, r, q, l$ calculated using Eqs.19, 22, 27 and 30.

\begin{tabular}{ccccc}
\hline \multirow{2}{*}{ Values of $p, r, q$ and $l$} & \multicolumn{3}{c}{ Values of $a$} & Eq.30 \\
\cline { 2 - 5 } & Eq.19 & $\mathbf{E q . 2 2}$ & 0 & 0.1910 \\
0 & 0 & -0.1950 & 0.0025 & 0.1901 \\
0.01 & -0.00246 & -0.1931 & 0.0463 & 0.1616 \\
0.5 & -0.0462 & -0.1520 & 0.0577 & 0.1240 \\
\hline
\end{tabular}

Table 2. Concentration of substrate $u(x)$ when $\gamma \beta \leq 1$ and $\gamma \beta \geq 1$. 
Table 3. Concentration of mediator $v(x)$ when $\gamma / \beta \leq 1$ and $\gamma / \beta \geq 1$.

\begin{tabular}{|c|c|c|c|c|c|}
\hline \multirow{2}{*}{ s.no } & \multirow{2}{*}{ Conditions } & \multicolumn{3}{|l|}{$v(x)$} & \multirow{2}{*}{ Figures } \\
\hline & & This work & & Lyons and Bartlett [2] & \\
\hline 1. & $\begin{array}{c}\gamma / \beta<<1 \\
\quad(\text { or) } \\
\gamma / \beta=q \leq 1\end{array}$ & $\begin{array}{l}\qquad v(x) \approx 1-4 a x+0.5 q\left(1+a-q-2 a q-a^{2} q\right) x^{2} \\
\text { where } \\
\qquad a=0.25 q-0.239 q^{2}-0.099 q^{3}+0.078 q^{4}\end{array}$ & (28) & ------- & $\begin{array}{c}\text { Figure } 5 \\
\gamma / \beta=0.01,0.25,0.5 \\
\text { Figure } 13 \\
\gamma / \beta=0.5\end{array}$ \\
\hline 2. & $\begin{array}{l}\gamma / \beta>1 \\
\quad(\text { or) } \\
\beta / \gamma=l \leq 1\end{array}$ & $\begin{array}{l}\qquad v(x) \approx 1-4 a x+0.5\left[1-l\left(1-a+a^{2}-a^{3}\right)\right] x^{2}+ \\
\text { where } \\
\qquad \begin{array}{l}\left.\quad-0.667 a+a l\left(1-a+a^{2}-a^{3}\right)\right] x^{3} \\
\quad \\
\quad+\left(1.831-2.996 a^{5} l+0.497 a^{4} l-1.998 a^{3} l\right. \\
\quad=0\end{array}\end{array}$ & (31) & $v(x)=\cosh (x)-\tanh \sinh (x)$ & $\begin{array}{c}\text { Figure } 6 \\
\gamma / \beta=2,4,100 \\
\text { Figure } 14 \\
\gamma / \beta=2\end{array}$ \\
\hline
\end{tabular}

Table 4. Current $I$ when $\gamma \beta \leq 1$ and $\gamma \beta \geq 1$.

\begin{tabular}{|c|c|c|c|c|c|c|}
\hline \multirow{2}{*}{ s.no } & \multirow{2}{*}{ Conditions } & \multicolumn{4}{|c|}{ Current $I$} & \multirow{2}{*}{ Figures } \\
\hline & & This work & & Lyons and Bartlett [2] & & \\
\hline 1. & $\begin{array}{c}\gamma \beta<<1 \\
\text { (or) } \\
\gamma \beta=p \leq 1\end{array}$ & $I=\left(1-0.367 a^{2}\right) p+\left(-0.533 a^{2}+1.333 a-1\right) p^{2}$ & (32) & $I=\sqrt{p}$ & (43) & $\begin{array}{l}\text { Figure } 7 \\
\text { Figure } 15\end{array}$ \\
\hline 2. & $\begin{array}{c}\gamma \beta>>1 \\
\text { (or) } \\
1 / \gamma \beta=r \leq 1\end{array}$ & $\begin{aligned} I= & 1-r+0.533 a^{2}+1.3332 a-1.3322 a r \\
& -0.499 a^{2} r-2.332 a^{3} r-2 a^{4} r\end{aligned}$ & (34) & $I=\tanh$ & (44) & $\begin{array}{l}\text { Figure } 8 \\
\text { Figure } 16\end{array}$ \\
\hline
\end{tabular}

Table 5. Current $I$ when $\gamma / \beta \leq 1$ and $\gamma / \beta \geq 1$.

\begin{tabular}{|c|c|c|c|c|c|c|}
\hline \multirow{2}{*}{ s.no } & \multirow{2}{*}{ Conditions } & \multicolumn{4}{|c|}{ Current $I$} & \multirow{2}{*}{ Figures } \\
\hline & & This work & \multicolumn{3}{|c|}{ Lyons and Bartlett [2] } & \\
\hline 1. & $\begin{array}{c}\gamma / \beta<<1 \\
\text { (or) } \\
\gamma / \beta=q \leq 1\end{array}$ & $I=4 a$ & (33) & $I=\sqrt{q}$ & (45) & $\begin{array}{c}\text { Figure } 9 \\
\text { Figure } 17\end{array}$ \\
\hline 2. & $\begin{array}{c}\gamma / \beta>1 \\
\text { (or) } \\
\beta / \gamma=l \leq 1\end{array}$ & $I=4 a$ & (35) & $I=\tanh$ & (46) & $\begin{array}{l}\text { Figure } 10 \\
\text { Figure } 18\end{array}$ \\
\hline
\end{tabular}

\section{COMPARISON WITH LYONS AND BARTLETT [2] WORK}

Lyons and Bartlett [2] takes the (8) in the form

$$
d^{2} u / d x^{2}-\gamma \beta(u v)^{1 / 2}=0
$$

when $\gamma \beta<<1$ whereas

$$
d^{2} u / d x^{2}-u=0
$$

when $\gamma \beta>>1$. The third term in the Eqs.16 and 17 is not found in the Eqs.36 and 37. When $\gamma<<\beta$ (9) takes the form:

$$
\frac{d^{2} v}{d x^{2}}-\frac{\gamma(u v)^{1 / 2}}{\beta}=0
$$


whereas when $\gamma>>\beta$

$$
d^{2} v / d x^{2}-v=0
$$

The third term in the Eqs.24 and 25 is not present in the Eqs.38 and 39. Figure 2 shows the schematic representation of the differential equations describing the transport and kinetics in a microheterogeneous system by (Lyons and Bartlett [2]). Each of these expressions represents the approximations to the set of master equations outlined in the Eqs.8 and 9. Lyons and Bartlett [2]) obtained the concentration of the substrate as

$$
\begin{aligned}
& u(x)=[1-0.2887 \sqrt{p}(1-x)]^{4} \text { if } p=\gamma \beta<<1 \\
& u(x)=\cosh (x) \sec h \quad \text { if } \quad p=\gamma \beta>>1
\end{aligned}
$$

Similarly, mediator concentration as

$$
v(x)=\cosh (x)-\tanh \sinh (x) \quad \text { if } l=\beta / \gamma<<1
$$

But a definite solution for mediator concentration is not arrived upon in the case of $q=\gamma / \beta<<1$. The Eqs.40 and 41 derived by Lyons and Bartlett [2] satisfy the boundary condition (11), but the Eq.40 does not satisfy the boundary condition (10). In the same way Eq.41 is independent of the parameter $1 / \gamma \beta$ whereas our Eqs.20 and 23 satisfy the boundary conditions (10) and (11). Similarly Eq.42 is independent of the parameter $\beta / \gamma$ whereas our Eqs.28 and $\mathbf{3 1}$ satisfy the boundary conditions (10) and (11). Lyons and Bartlett [2] obtained the corresponding dimensionless current $I$ as follows.

$$
I=\sqrt{p} \quad \text { if } \quad p=\gamma \beta<<1
$$

$$
\begin{array}{llr}
I=\tanh & \text { if } \quad r=1 / \gamma \beta<1 \\
I=\sqrt{q} & \text { if } q=\gamma / \beta<1 \\
I=\tanh & \text { if } \quad l=\beta / \gamma<<1
\end{array}
$$

Eqs.44 and 46 are independent of the parameters $1 / \gamma \beta$ and $\beta / \gamma$ whereas our Eqs.34 and 35 depend on the parameters $1 / \gamma \beta$ and $\beta / \gamma$.

\section{DISCUSSION OF STEADY STATE PROBLEM}

The comparison of concentration of substrate $u(x)$ between the Eqs.20 and 23 (This work) and Eqs.40 and 41 (Lyons and Bartlett [2]) are represented in Figure 3, Figure 4 for various values of $\gamma \beta$. From these Figures it is understood that the value of the concentration decreases when $\gamma \beta$ increases. Concentration is slowly increasing when $x \geq 0.5$ for all values of $\gamma \beta$. Then the concentration of $u(x)$ becomes 1 when $x=1$ for all values of $\gamma \beta$. The comparison of concentration mediator $v(x)$ between the Eqs.28 and 31 (This work) and Eq.42 (Lyons and Bartlett [2]) are represented in Figure 5 and Figure 6 for various values of $\gamma / \beta$. From these figures, it is deducted that the value of the concentration of $u(x)$ decreases when $\gamma / \beta$ increases. Concentration is slowly decreasing when $x \geq 0.6$ for all values of $\gamma / \beta$. From these Figures 3-6, it is constructed that Eqs.20, 23, 28 and 31 satisfy their boundary conditions (10) and (11).

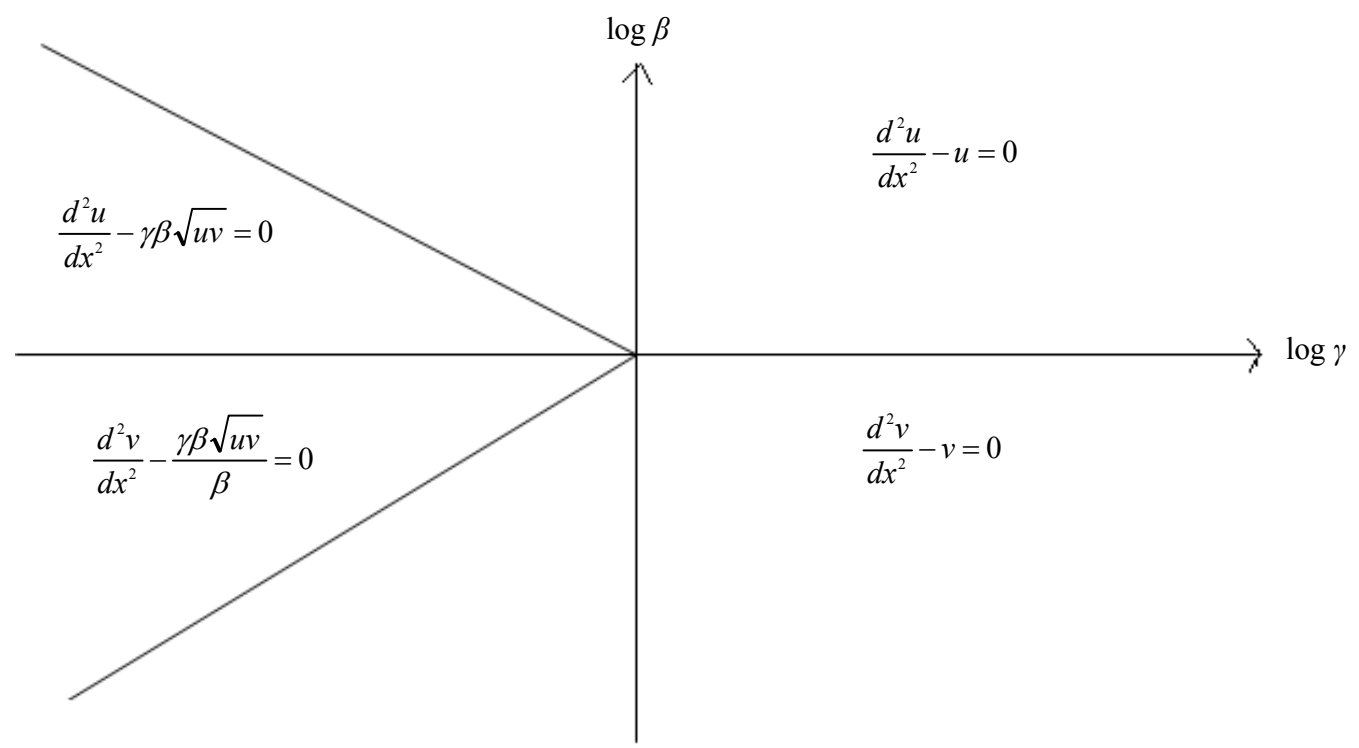

Figure 2. Schematic representation of the differential equations describing the transport and kinetics in a microheterogeneous system by Lyons and Bartlett [2]. Each of these expressions represents approximations to the set of master equations outlined in the Eqs.8 and 9. 


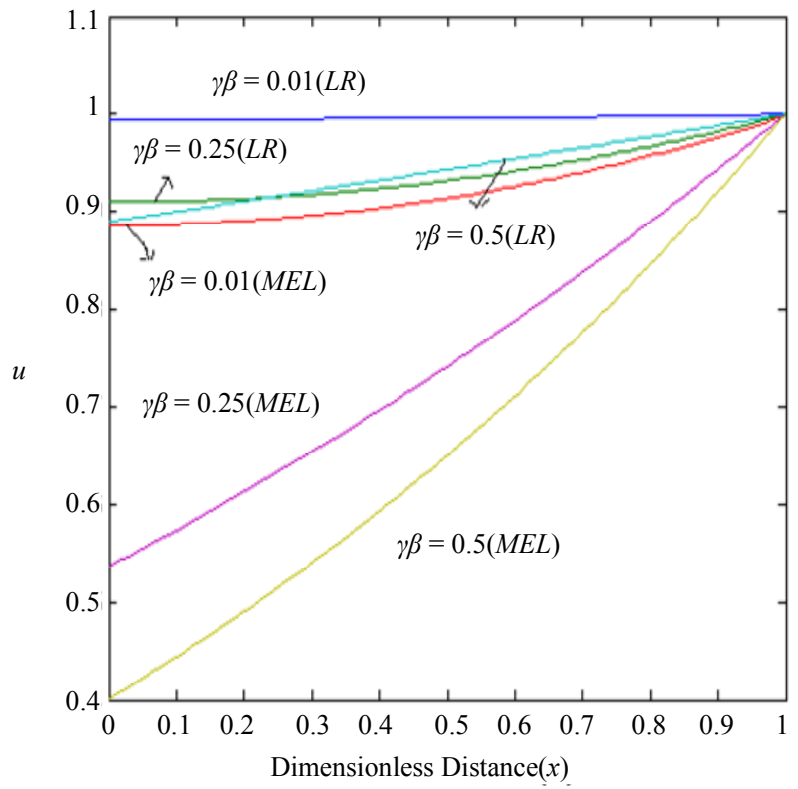

Figure 3. Profiles of the dimensionless substrate concentration $u$, for various values of $\gamma \beta$ when $\gamma \beta<<1$. The curve is plotted using Eqs.20 and 40.

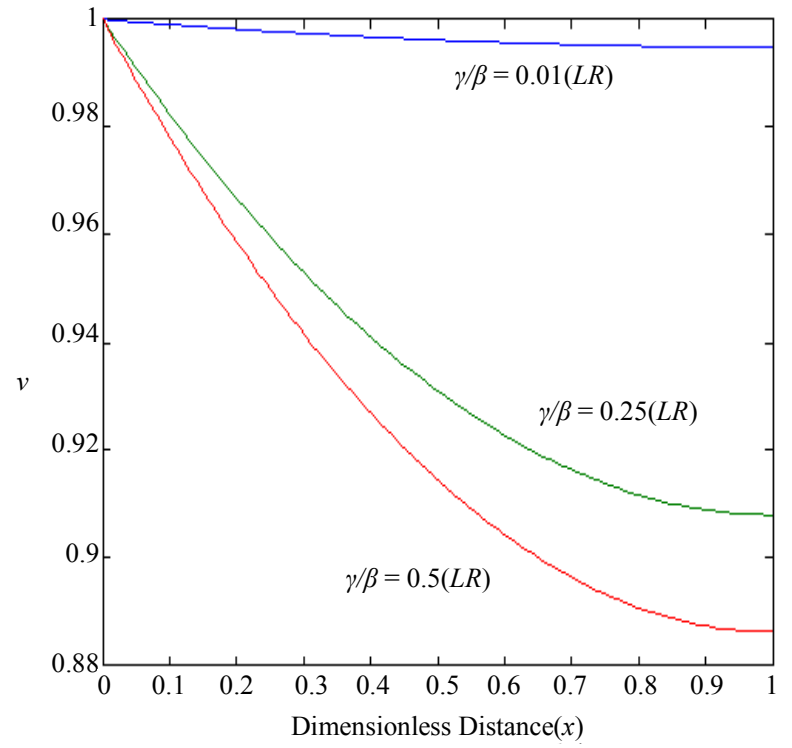

Figure 5. Profiles of the dimensionless mediator concentration $v$, for various values of $\gamma / \beta$ when $\gamma / \beta<<1$. The curve is plotted using Eq.28.

Figure 7 shows the comparison of dimensionless current $I$ between the Eqs.32 and $\mathbf{4 3}$ for various values of $\gamma \beta$ when $\gamma \beta<<1$. Figure 8 shows the comparison of dimensionless current $I$ between the Eqs.34 and $\mathbf{4 4}$ for various values of $\gamma \beta$ when $\gamma \beta>>1$. Figure 9 shows the comparison of dimensionless current $I$ between the Eqs.33

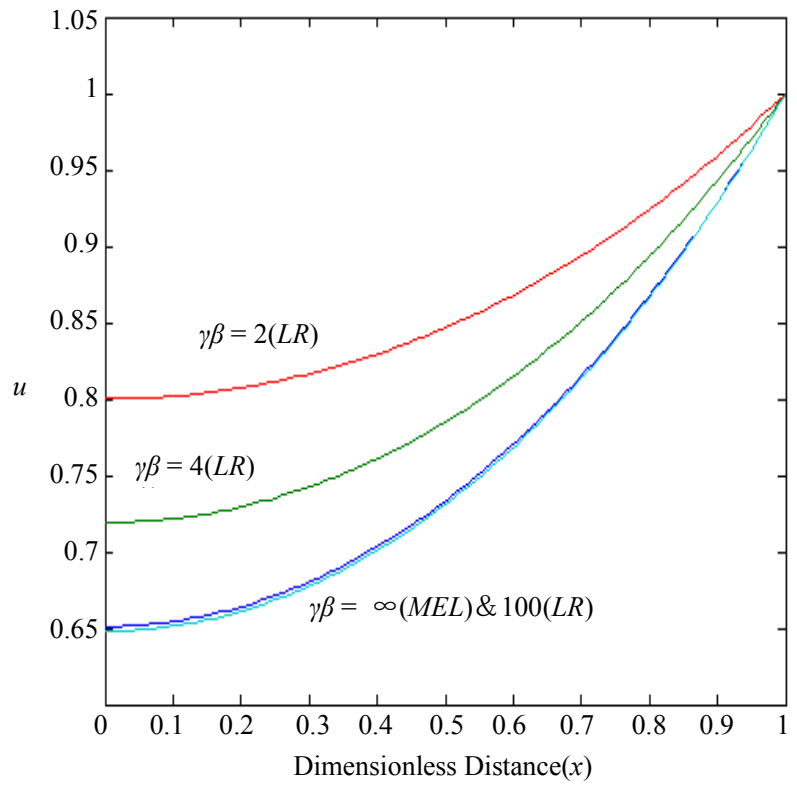

Figure 4. Profiles of the dimensionless substrate concentration $u$, for various values of $\gamma \beta$ when $\gamma \beta>>1$. The curve is plotted using Eqs.23 and 41.

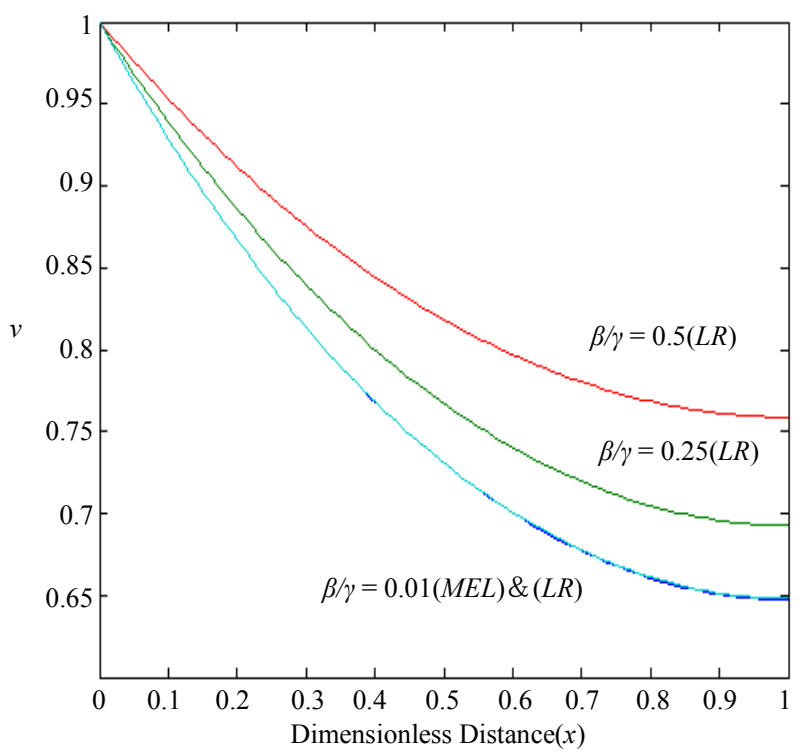

Figure 6. Profiles of the dimensionless mediator concentration $v$, for various values of $\gamma / \beta$ when $\gamma / \beta>>1$. The curve is plotted using Eqs.31 and $\mathbf{4 2}$.

and 45 for various values of $\gamma / \beta$ when $\gamma / \beta<<1$. Figure 10 shows the comparison of dimensionless current $I$ between the Eqs.35 and $\mathbf{4 6}$ for various values of $\gamma / \beta$ when $\gamma / \beta>>1$. In all case diagrams as shown in figures, there is a vast variation in the current curves.

Figures 11 and 12 show the comparison of our di- 
mensionless concentration $u$ evaluated using Eqs.20 and 23 (This work) together with the simulation results (This work) and Eqs.40 and 41 (Lyons and Bartlett [2]) for the case of $\gamma \beta<<1$ and $\gamma \beta>>1$. Figures 13 and 14 indicate the comparison of our dimensionless concentration $v$ calculated using Eqs.28 and 31 (This work) together with the simulation results (This work) and Eq.42 (Lyons and Bartlett [2]) for the case of $\gamma / \beta<<1$ and $\gamma / \beta>>1$. In all cases, there is a good match between our analytical and simulation results.

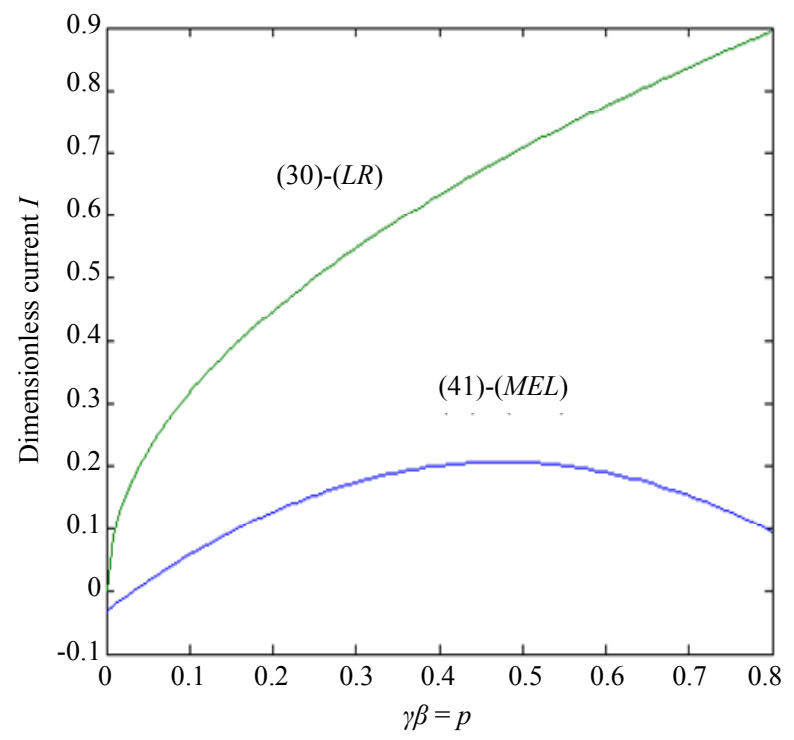

Figure 7. Plot of dimensionless current versus $\gamma \beta=p$. Current is calculated in the Eqs.32 and $\mathbf{4 3}$.

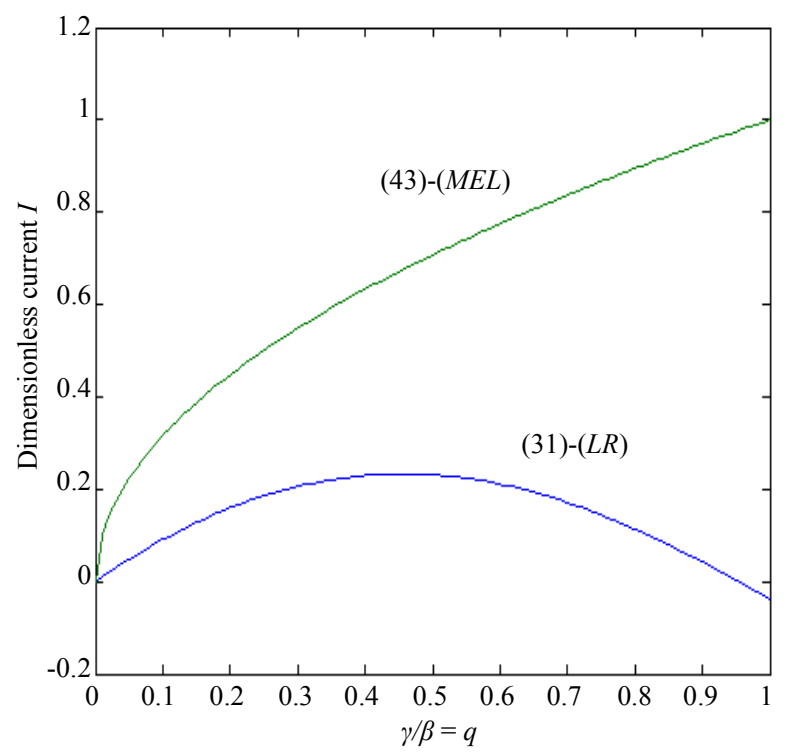

Figure 9. Plot of dimensionless current versus $\gamma / \beta=q$. Current is calculated in the Eqs.33 and $\mathbf{4 5 .}$
Figures 15 and 16 show the comparison of our dimensionless current $I$ versus $\gamma \beta$ evaluated using Eqs.32 and 34 (This work) together with the simulation results (This work) and Eqs.43 and 44 (Lyons and Bartlett [2]) for the case of $\gamma \beta<<1$ and $\gamma \beta>>1$. Figures 17 and 18 indicate the comparison of our dimensionless current $I$ versus $\gamma / \beta$ calculated using Eqs.33 and 35 (This work) together with the simulation results (This work) and Eqs.45 and 46 (Lyons and Bartlett [2]) for the case of $\gamma / \beta<<1$ and $\gamma / \beta>>1$. In all cases, there is a good

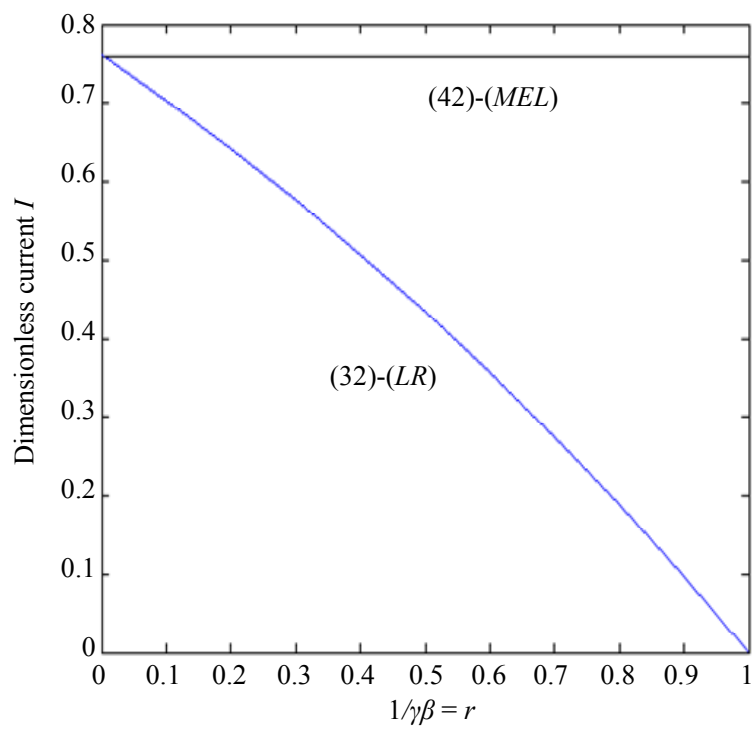

Figure 8. Plot of dimensionless current versus $1 / \gamma \beta=r$. Current is calculated in the Eqs.34 and $\mathbf{4 4 .}$

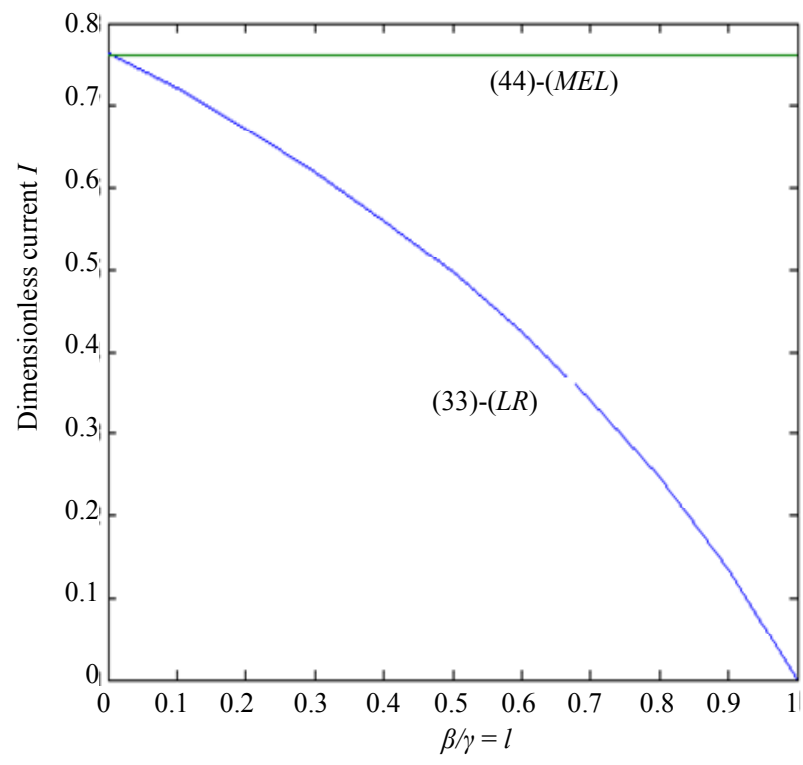

Figure 10. Plot of dimensionless current versus $\beta / \gamma=l$. Current is calculated in the Eqs.35 and $\mathbf{4 6 .}$ 


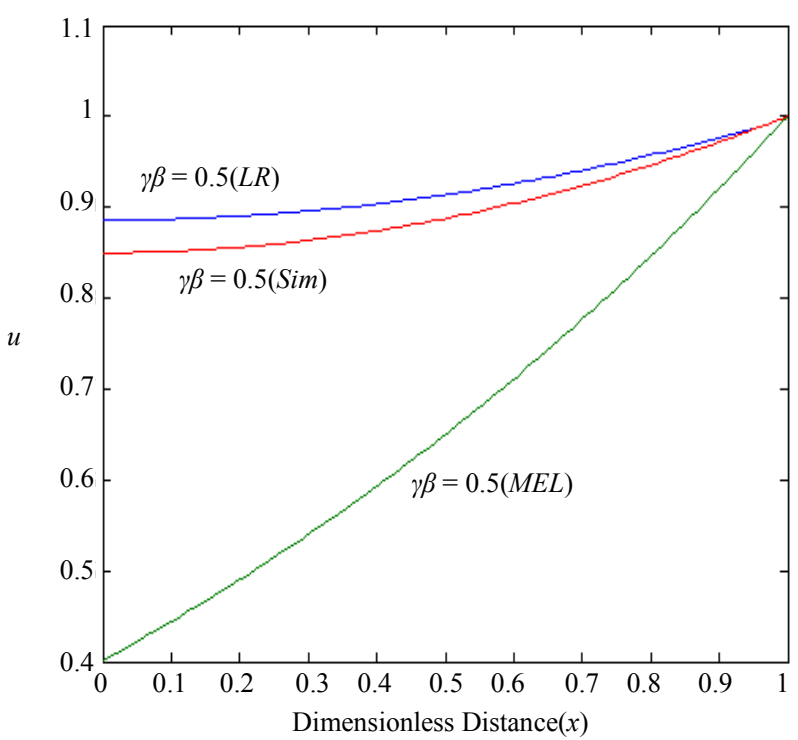

Figure 11. Comparison of our dimensionless concentration $u$ using Eq.20 (This work), Eq.40 (Lyons and Bartlett [2]) and simulation results using Scilab ( This work). Here the value of $\gamma \beta=0.5<<1$.

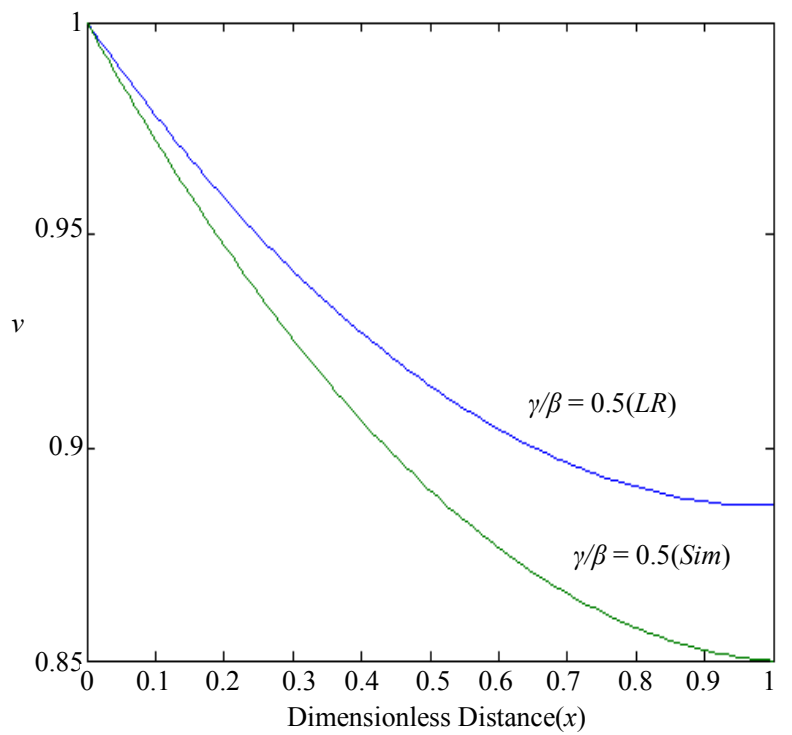

Figure 13. Comparison of our dimensionless concentration $v$ using Eq.28 (This work) and simulation results using Scilab (This work). Here the value of $\gamma / \beta=0.5<<1$.

match between our analytical and simulation results.

\section{MATHMATICAL FORMULATION OF THE PROBLEM AND ANALYSIS (NON-STEADY STATE)}

The initial boundary value problems which has to be solved for the case of non-steady state can be written in

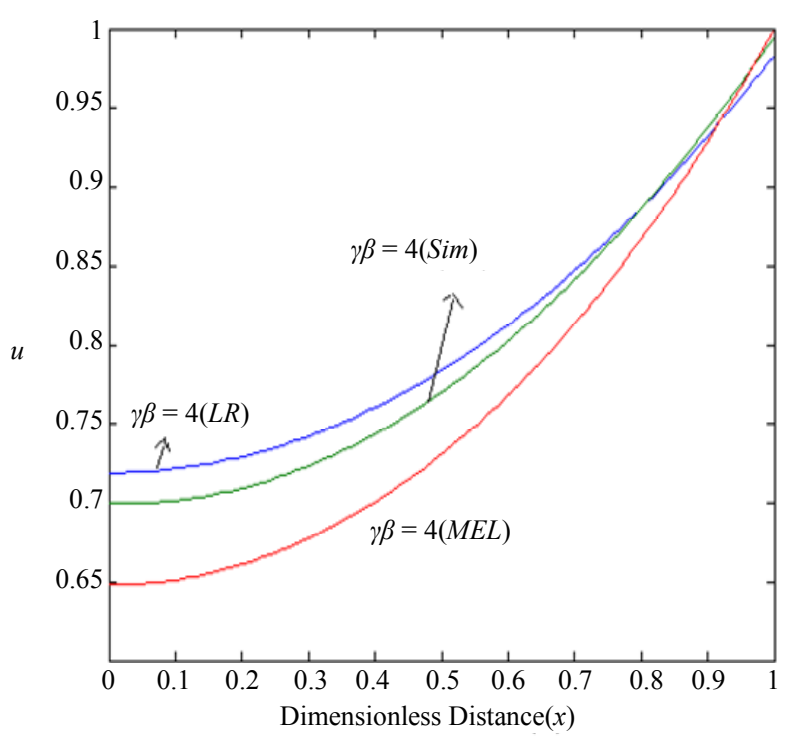

Figure 12. Comparison of our dimensionless concentration $u$ using Eq.23 (This work), Eq.41 (Lyons and Bartlett [2]) and simulation results using Scilab (This work). Here the value of $\gamma \beta=4>>1$.

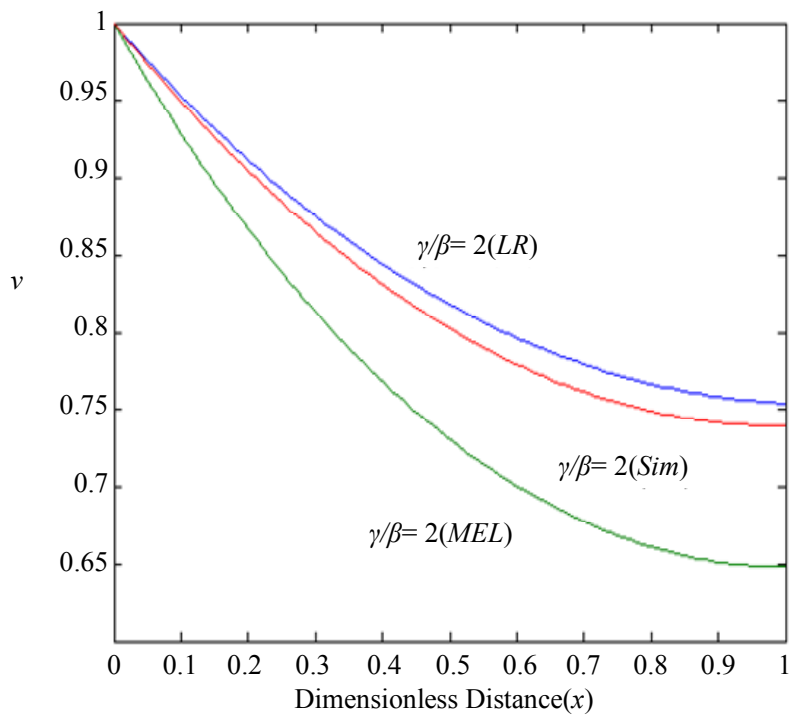

Figure 14. Comparison of our dimensionless concentration $v$ using Eq.31 (This work), Eq.42 (Lyons and Bartlett [2]) and simulation results using Scilab (This work). Here the value of $\gamma / \beta=2>>1$.

dimensionless form as follows

$$
\frac{d u}{d t}=\frac{d^{2} u}{d x^{2}}-\frac{\gamma \beta(u v)^{1 / 2}}{1+\gamma \beta(v / u)^{1 / 2}}
$$

for the substrate, and

$$
\frac{d v}{d t}=\frac{d^{2} v}{d x^{2}}-\frac{\gamma(u v)^{1 / 2}}{\beta+\gamma(u / v)^{1 / 2}}
$$




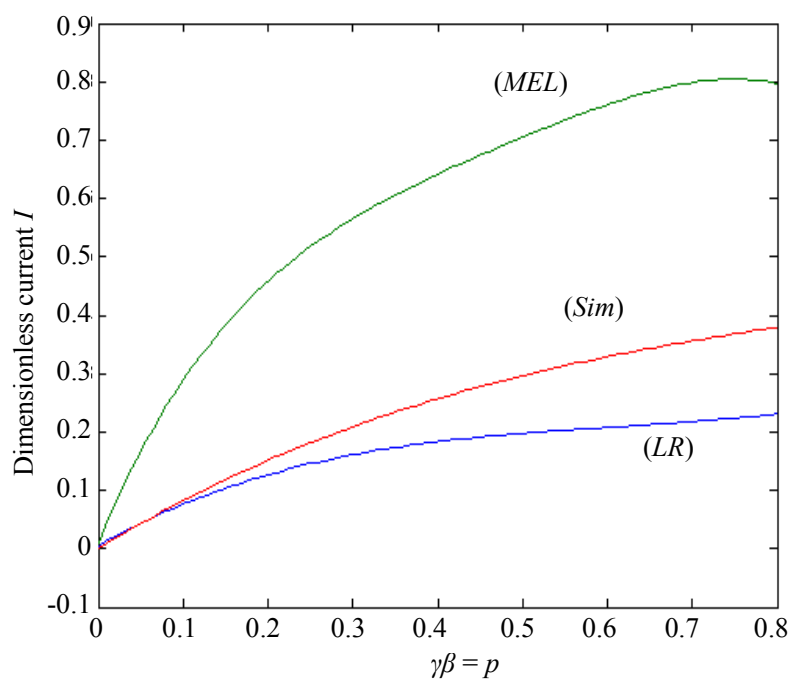

Figure 15. Comparison of our dimensionless current $I$ versus $\gamma \beta=p$ using Eq.32 (This work), Eq.43 (Lyons and Bartlett [2]) and simulation results using Scilab (This work). Here the value of $\gamma \beta<<1$

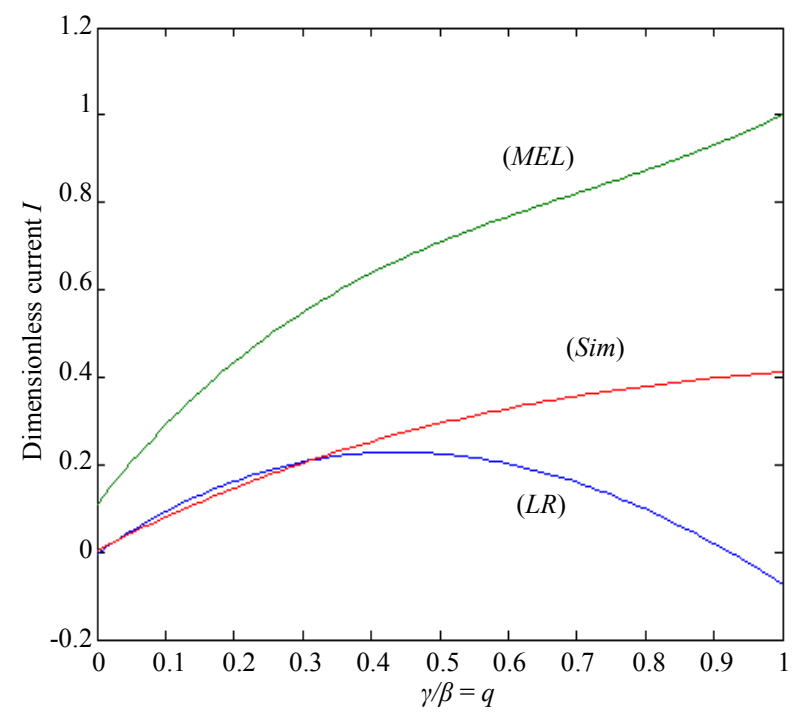

Figure 17. Comparison of our dimensionless current $I$ versus $\gamma / \beta=q$ using Eq.33 (This work), Eq.45 (Lyons and Bartlett [2]) and simulation results using Scilab (This work). Here the value of $\gamma / \beta<<1$.

for the mediator respectively. These equations must obey the following initial and boundary conditions

$$
\begin{aligned}
& t=0, u=1 \text { and } v=0.001 \\
& x=0, v=1 \text { and } d u / d x=0 \\
& x=1, d v / d x=0 \text { and } u=1
\end{aligned}
$$

To my knowledge no rigorous analytical (or) numerical solutions for the transient problems have been reported. Numerical simulation of substrate concentration

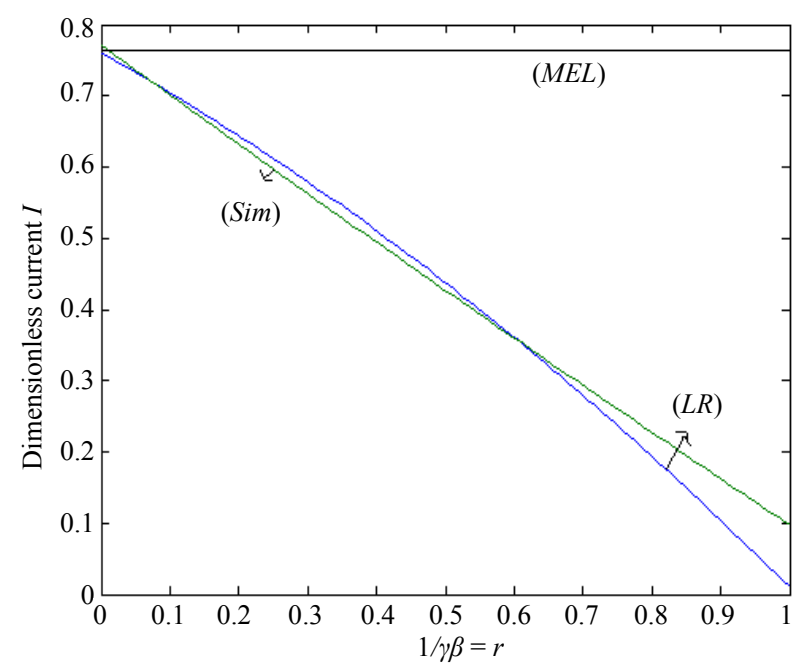

Figure 16. Comparison of our dimensionless current $I$ versus $1 / \gamma \beta=r$ using Eq.34 (This work), Eq.44 (Lyons and Bartlett [2]) and simulation results using Scilab (This work). Here the value of $\gamma \beta>>1$.

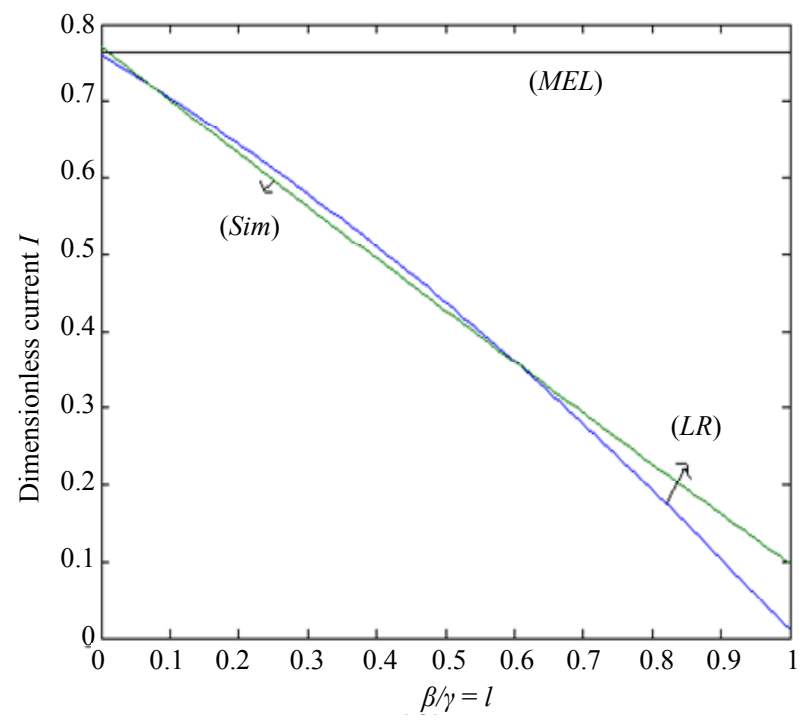

Figure 18. Comparison of our dimensionless current $I$ versus $\beta / \gamma=l$ using Eq.35 (This work), Eq.46 (Lyons and Bartlett [2]) and simulation results using Scilab (This work). Here the value of $\gamma / \beta>>1$.

and mediator concentration can be evaluated using Scilab software (Appendix-B) .

\section{DISCUSSION OF NON-STEADY STATE PROBLEM}

The normalized numerical simulations of three dimensional substrate concentration $u(x)$ is shown in Figure 19, Figure 21 and Figure 23. As shown in Figures 19, 


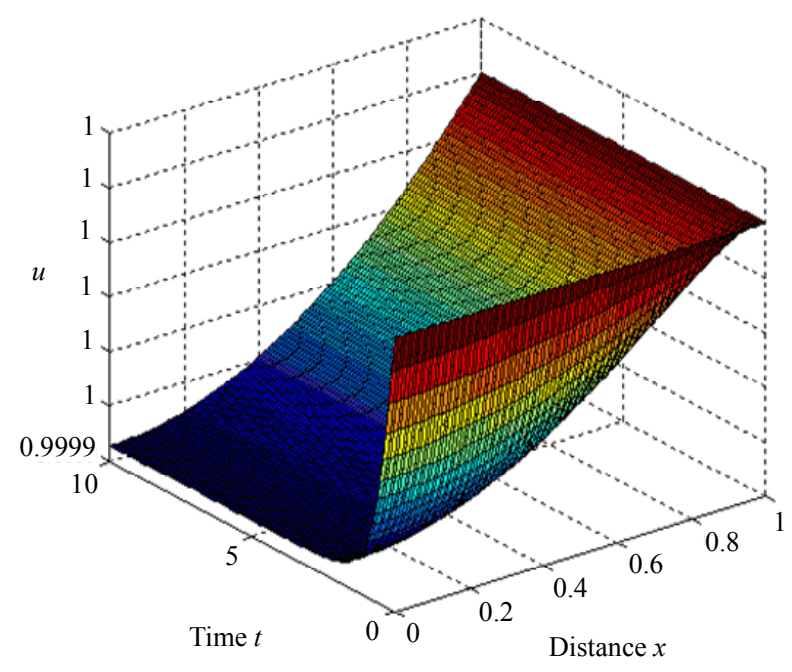

Figure 19. The normalized numerical simulation of three dimensional substrate concentration $u(x)$. The plot was constructed using Eq.47 for $\gamma=0.01, \beta=0.01$.

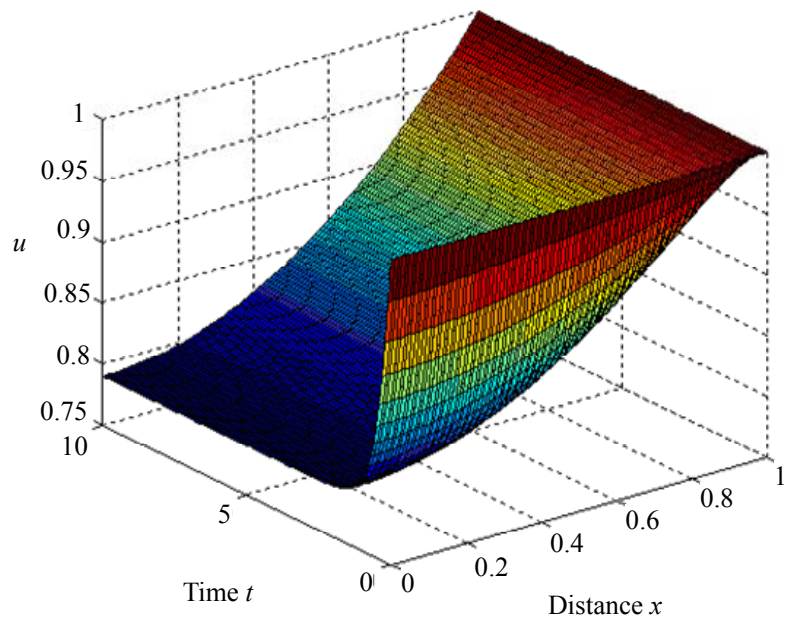

Figure 21. The normalized numerical simulation of three dimensional substrate concentration $u(x)$. The plot was constructed using Eq.47 for $\gamma=1, \beta=1$.

Figure 21 and Figure 23 give the calculated response curve at different $\gamma$ and $\beta$ values in our diagrams. The time dependent concentration $u(x)$ using Eq.47 is represented in Figure 19, Figure 21 and Figure23 for various values of $\gamma$ and $\beta$. Concentration is slowly decreasing when $\gamma$ and $\beta$ is increasing. Then the concentration of $u(x)=1$ when $x=1$ and also for all values of $\gamma, \beta$ and $t$. The normalized numerical simulation of three dimensional mediator concentration $v(x)$ is shown in Figure 20, Figure 22 and Figure 24. These figures show the calculated curve at different $\gamma$ and $\beta$ values in our diagrams. The time dependent curve $v(x)$ using Eq.48 is represented in Figure 20, Fi-

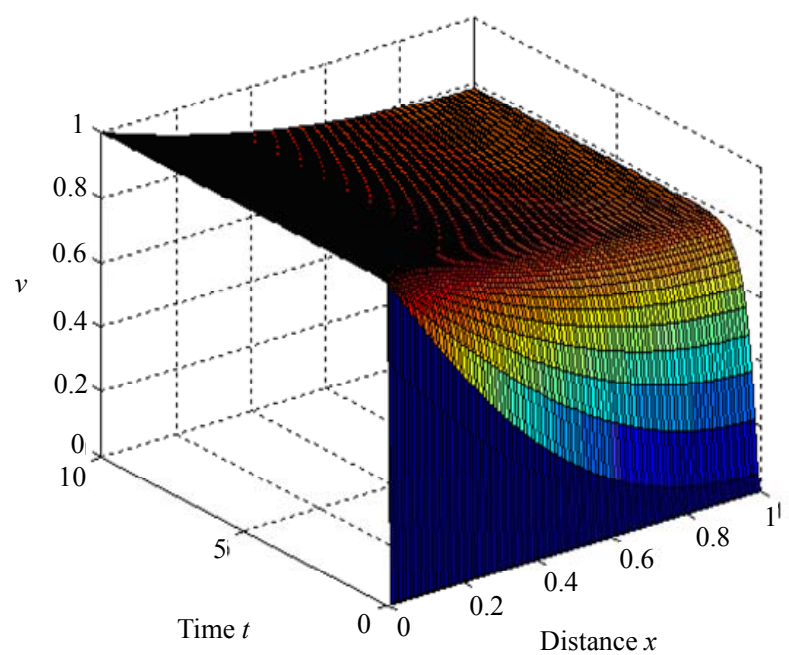

Figure 20. The normalized numerical simulation of three dimensional mediator concentration $v(x)$. The plot was constructed using Eq.48 for $\gamma=0.01, \beta=0.01$.

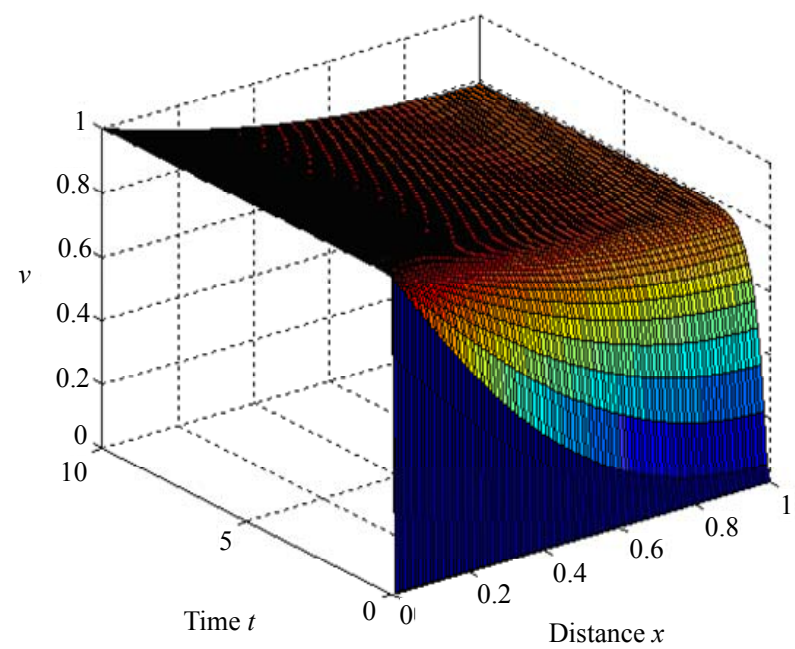

Figure 22. The normalized numerical simulation of three dimensional mediator concentration $v(x)$. The plot was constructed using Eq.48 for $\gamma=1, \beta=1$.

gure 22 and Figure 24 for all values of $\gamma, \beta$ and $t \cdot \gamma$ and $\beta$ larger than 1 may be appropriate because the slope of the curves corresponding to $\gamma \geq 1$ and $\beta \geq 1$ are almost identical although the analytical ranges are different. The slope of the curves decreases dramatically and the concentration is identical range when $\gamma$ and $\beta$ is high.

\section{CONCLUSIONS}

We have presented a simple analysis of reaction/diffusion within a conducting polymer film which is deposited on a microelectrode. The transport and kinetics are quantified in terms of a fundamental reaction/diffusion 


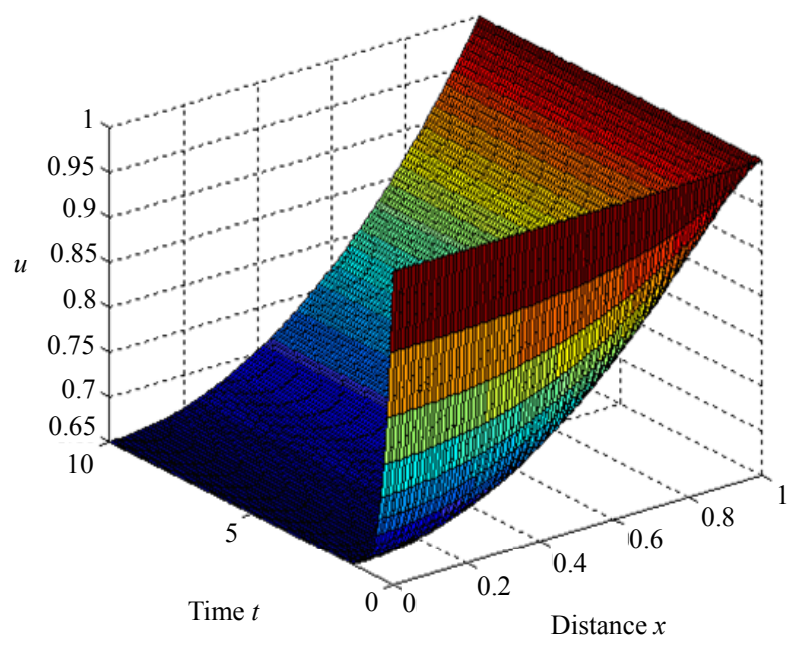

Figure 23. The normalized numerical simulation of three dimensional substrate concentration $u(x)$. The plot was constructed using Eq.47 for $\gamma=10, \beta=10$.

parameters $\gamma \beta, \gamma / \beta$ and the analytical expression of the substrate concentration and mediator concentration within the polymer film are thus derived. An analytical expression for the steady state current response is also presented. A non linear time independent partial differential equation has been formulated and solved using He's variational iteration method. The primary result of this work is first approximate calculation of substrate concentration and mediator concentration for all values of $\gamma \beta$ and $\gamma / \beta$. It gives good agreement with previous published limiting case results. The extension of the procedure to other two-dimensional and three-dimensional geometries with various complex boundary conditions seems possible.

\section{ACKNOWLEDGEMENTS}

This work was supported by the Department of Science and Technology (DST) Government of India. The authors also thank Mr. M. S. Meenakshisundaram, Secretary, The Madura College Board Dr. T. V. Krishnamoorthy, Principal, and Mr. S. Thiagarajan Head of the Department of Mathematics, The Madura College, Madurai, for their constant encouragement. It is our pleasure to thank the referees for their valuable comments.

\section{REFERENCES}

[1] Lyons, M.E.G., McCormack, D.E. and Bartlett, P.N. (1989) Microheterogeneous catalysis in modified electrodes. Journal of Electroanalytical Chemistry, 261(1), 51-59.

[2] Lyons, M.E.G. and Bartlett, P.N. (1991) Microheterogeneous catalysis in modified electrodes: Part 2. Electron transfer mediator/catalyst composites. Journal of Electroanalytical Chemistry, 316(1-2), 1-22.

[3] Harrison, D.J. and Wrighton, M.S. (1984) Catalysis of

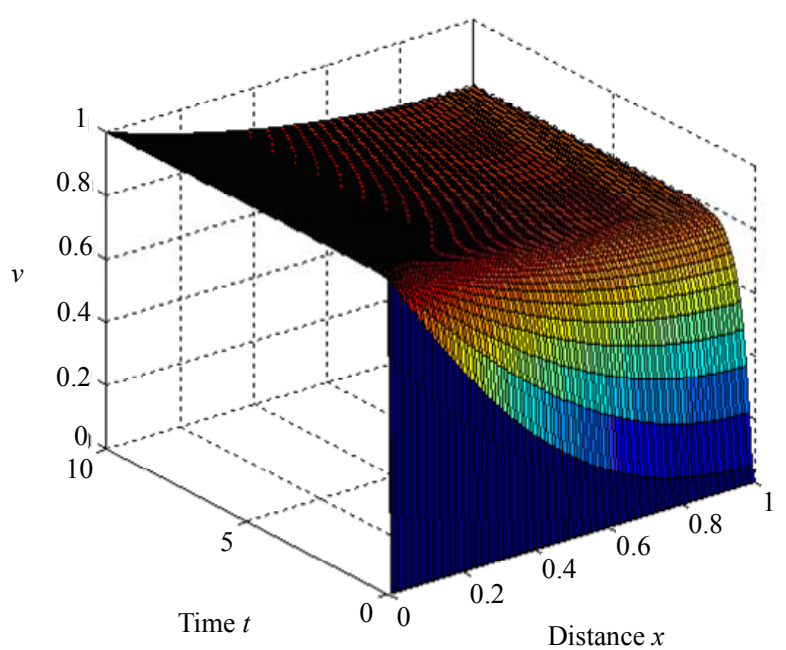

Figure 24. The normalized numerical simulation of three dimensional mediator concentration $v(x)$. The plot was constructed using Eq.48 for $\gamma=10, \beta=10$.

hydrogen evolution via deposition of palladium onto electrodes modified with an N, N'-Dialkyl-4, 4'-bipyridinium-based polymer: Dependence of rate on palladium coverage. Journal of Physical Chemistry, 88(18), 39323935.

[4] Andrieux, C.P., Dumas-Bouchiat, J.M. and Saveant, J.M. (1982) Catalysis of electrochemical reactions at redox polymer electrodes: Kinetic model for stationary voltammetric techniques. Journal of Electroanalytical Chemistry, 131, 1-35.

[5] Albery, W.J. and Hillman, A.R. (1984) Transport and kinetics in modified electrodes. Journal of Electroanalytical Chemistry, 170(1-2), 27-49.

[6] Hillman, A.R. and Linford, R.G. (1987) Electrochemical science and technology of polymers. Elsevier, Amsterdam, 1, 103-291.

[7] Albery, W.J. and Hillman, A.R. (1981) Modified electrodes. Annual Report Section C of the Royal Society of Chemistry, 78, 377-438.

[8] He, J.H. (1999) Variational iteration method-a kind of nonlinear analytical technique: Some examples. International Journal of Non-Linear Mechanics, 34(4), 699-708.

[9] Momani, S. and Abuasad, S. (2006) Application of He's variational iteration method to Helmholtz equation. Chaos, Solitons \& Fractals, 27(5), 1119-1123.

[10] Abdou, M.A. and Soliman, A.A. (2005) Variational iteration method for solving Burger's and coupled Burger's equations. Journal of Computational and Applied Mathematics, 181(2), 245-251.

[11] He, J.H. and Wu, X.H. (2006) Construction of solitary solution and compacton-like solution by variational iteration method. Chaos, Solitons \& Fractals, 29(1), 108.

[12] Rajendran, L. and Rahamathunissa, G. (2008) Application of He's variational iteration method in nonlinear boundary value problems in enzyme-substrate reaction diffusion processes: Part 1. The steady-state amperometric response. Journal of Mathematical Chemistry, 44(3), 849-861. 


\section{APPENDIX A}

In this appendix, we derive the general solution of nonlinear reaction (8), (9), (10), (11) using He's variational iteration method. To illustrate the basic concepts of variational iteration method (VIM), we consider the following non-linear partial differential equation [8-12]

$$
L[u(x)]+N[u(x)]=g(x)
$$

where $L$ is a linear operator, $N$ is a non-linear operator, and $g(x)$ is a given continuous function. According to the variational iteration method, we can construct a correct functional as follows [11]

$$
u_{n+1}(x)=u_{n}(x)+\int_{0}^{x} \lambda\left[L\left[u_{n}(\tau)\right]+N\left[\tilde{u_{n}}(\tau)\right]-g(\tau)\right] d \tau
$$

Where $\lambda$ is a general Lagrange multiplier which can be identified optimally via variational theory, $u_{n}$ is the $\mathrm{n}^{\text {th }}$ approximate solution, and $u_{n}$ denotes a restricted variation, i.e., $\delta u_{n}=0$. In this method, a trial function (an initial solution) is chosen which satisfies given boundary conditions. Using the above variational iteration method we can write the correction functional of Eqs.16, 17, 24 and 25 as follows

$$
\begin{aligned}
& u_{n+1}(x)=u_{n}(x)+\int_{0}^{x} \lambda[u_{n}^{\prime \prime}(\xi)+\overbrace{\gamma^{2} \beta^{2} v_{n}(\xi)}^{\sim}-\overbrace{\left.\gamma \beta u_{n}{ }^{1 / 2}(\xi) v_{n}{ }^{1 / 2}(\xi)\right)}^{\sim}] d \xi \\
& v_{n+1}(x)=v_{n}(x)+\int_{0}^{x} \lambda[v_{n}^{\prime \prime}(\xi)-\overbrace{\frac{\gamma}{\beta} u_{n}{ }^{1 / 2}(\xi) v_{n}{ }^{1 / 2}(\xi)}^{\sim}+\overbrace{\frac{\gamma^{2}}{\beta^{2}} u_{n}(\xi)}^{\sim}] d \xi \\
& u_{n+1}(x)=u_{n}(x)+\int_{0}^{x} \lambda[u_{n}^{\prime \prime}(\xi)-u_{n}(\xi)+\overbrace{\frac{1}{\gamma \beta} u_{n}^{1 / 2}(\xi) v_{n}^{-1 / 2}(\xi)}^{\sim}] d \xi \\
& v_{n+1}(x)=v_{n}(x)+\int_{0}^{x} \lambda[v_{n}^{\prime \prime}(\xi)-v_{n}(\xi)+\overbrace{\frac{\beta}{\gamma} \frac{v_{n}^{3 / 2}(\xi)}{u_{n}^{1 / 2}(\xi)}}^{\sim}] d \xi
\end{aligned}
$$

Taking variation with respect to the independent variables $u_{n}$ and $v_{n}$, we get

$$
\begin{aligned}
& \delta u_{n+1}(x)=\delta u_{n}(x)+\delta \int_{0}^{x} \lambda[u_{n}^{\prime \prime}(\xi)+\overbrace{\gamma^{2} \beta^{2} v_{n}(\xi)}^{\sim}-\overbrace{\left.\gamma \beta u_{n}^{1 / 2}(\xi) v_{n}^{1 / 2}(\xi)\right)}^{\sim}] d \xi \\
& \delta v_{n+1}(x)=\delta v_{n}(x)+\delta \int_{0}^{x} \lambda[v_{n}^{\prime \prime}(\xi)-\overbrace{\frac{\gamma}{\beta} u_{n}^{1 / 2}(\xi) v_{n}^{1 / 2}(\xi)}^{\sim}+\overbrace{\frac{\gamma^{2}}{\beta^{2}} u_{n}(\xi)}^{\sim}] d \xi \\
& \delta u_{n+1}(x)=\delta u_{n}(x)+\delta \int_{0}^{x} \lambda[u_{n}^{\prime \prime}(\xi)-u_{n}(\xi)+\overbrace{\frac{1}{\gamma \beta} u_{n}^{1 / 2}(\xi) v_{n}^{-1 / 2}(\xi)}^{\sim}] \\
& \delta v_{n+1}(x)=\delta v_{n}(x)+\delta \int_{0}^{x} \lambda[v_{n}^{\prime \prime}(\xi)-v_{n}(\xi)+\overbrace{\frac{\beta}{\gamma} \frac{v_{n}^{3 / 2}(\xi)}{u_{n}{ }^{1 / 2}(\xi)}}^{\tilde{y}}] d \xi
\end{aligned}
$$

where $\lambda$ is general Lagrangian multipliers, $u_{0}$ and $v_{0}$ are 
initial approximations or trial functions $\overbrace{\gamma^{2} \beta^{2} v_{n}(\xi)}^{\sim}$,

$$
\overbrace{\left.\gamma \beta u_{n}^{1 / 2}(\xi) v_{n}^{1 / 2}(\xi)\right)}^{\sim}, \overbrace{\frac{\gamma}{\beta} u_{n}^{1 / 2}(\xi) v_{n}^{1 / 2}(\xi)}^{\sim}, \overbrace{\frac{\gamma^{2}}{\beta^{2}} u_{n}(\xi)}^{\sim}
$$

$\overbrace{\frac{1}{\gamma \beta} u_{n}^{1 / 2}(\xi) v_{n}{ }^{-1 / 2}(\xi)}, \overbrace{\frac{\beta}{\gamma} \frac{v_{n}^{3 / 2}(\xi)}{u_{n}{ }^{1 / 2}(\xi)}}^{\sim}$ and are considered as restricted variations i.e. $\delta \widetilde{u}_{n}=0, \delta \widetilde{v}_{n}=0$ and $\delta \widetilde{u}_{n} \widetilde{v}_{n}=0$. Making the above correction functional (A7) to (A10) stationary, noticing that $\delta u_{n}(0)=0, \delta v_{n}(0)=0$ and $\delta u_{n}(0) v_{n}(0)=0$.

$$
\begin{aligned}
& \delta u_{n}: 1-\left.\lambda^{\prime}(\xi)\right|_{\xi=x}=0, \delta v_{n}: 1-\left.\lambda^{\prime}(\xi)\right|_{\xi=\tau}=0 \\
& \delta u_{n}^{\prime}:\left.\lambda(\xi)\right|_{\xi=x}=0, \quad \delta v_{n}^{\prime}:\left.\lambda(\xi)\right|_{\xi=x}=0 \\
& \delta u_{n}:\left.\lambda^{\prime \prime}(\xi)\right|_{\xi=x}=0, \quad \delta v_{n}:\left.\lambda^{\prime \prime}(\xi)\right|_{\xi=x}=0
\end{aligned}
$$

The above equations are called Lagrange-Euler equations. By solving the above equations the Lagrange multipliers, can be identified as

$$
\lambda(\xi)=(\xi-x)
$$

substituting the Lagrangian multipliers and $n=0$ in the iteration formula (A3, A4, A5, A6) we obtain,

$$
\begin{aligned}
& u_{1}(x)=u_{0}(x)+\int_{0}^{x}(\xi-x)\left[u_{0}^{\prime \prime}(\xi)-\gamma \beta u_{0}^{1 / 2}(\xi) v_{0}^{1 / 2}(\xi)+\gamma^{2} \beta^{2} v_{0}(\xi)\right] d \xi \\
& u_{1}(x)=u_{0}(x)+\int_{0}^{x}(\xi-x)\left[u_{0}^{\prime \prime}(\xi)-u_{0}(\xi)+(1 / \gamma \beta) u_{0}^{1 / 2}(\xi) v_{0}^{-1 / 2}(\xi)\right] d \xi \\
& v_{1}(x)=v_{0}(x)+\int_{0}^{x}(\xi-x)\left[v_{0}^{\prime \prime}(\xi)-(\gamma / \beta) u_{0}^{1 / 2}(\xi) v_{0}^{1 / 2}(\xi)+\gamma^{2} / \beta^{2} u_{0}(\xi)\right] d \xi \\
& v_{1}(x)=v_{0}(x)+\int_{0}^{x}(\xi-x)\left[v_{0}^{\prime \prime}(\xi)-v_{0}(\xi)+(\beta / \gamma) v_{0}^{3 / 2}(\xi) u_{0}^{-1 / 2}(\xi)\right] d \xi
\end{aligned}
$$

Assuming that its initial approximate solutions which satisfies the boundary conditions (10), (11) have the form

$$
u_{0}(x)=\left[1+a\left(1-x^{2}\right)\right]^{2}
$$

\section{APPENDIX B}

Scilab Program to find a solution of the Eqs.47-51.

function pdex 4

$\mathrm{m}=0$;

$\mathrm{x}=$ linspace $(0,1)$;

$\mathrm{t}=$ linspace $(0,1)$;

sol=pdepe(m,@pdex4pde,@pdex4ic,@pdex4bc,x,t);

$\mathrm{u} 1=\operatorname{sol}(:,:, 1)$;

$\mathrm{u} 2=\operatorname{sol}(:,:, 2)$;

figure

$\operatorname{surf}(\mathrm{x}, \mathrm{t}, \mathrm{u} 1)$

title('u1 $(\mathrm{x}, \mathrm{t})$ ')

xlabel('Distance $\mathrm{x}$ ')

ylabel('Time t')

figure

$\operatorname{surf}(\mathrm{x}, \mathrm{t}, \mathrm{u} 2)$

title('u2(x,t)')

xlabel('Distance $\mathrm{x}$ ')

ylabel('Time t')

$\%$

function $[\mathrm{c}, \mathrm{f}, \mathrm{s}]=\operatorname{pdex} 4 \mathrm{pde}(\mathrm{x}, \mathrm{t}, \mathrm{u}, \mathrm{DuDx})$

$$
v_{0}(x)=\left[1-2 a x+a x^{2}\right]^{2}
$$

By the iteration formula (A15) to (A18) we have the Eqs.18, 21, 26 and 29 in the text.

$$
\begin{aligned}
& \mathrm{c}=[1 ; 1] \\
& \mathrm{f}=[1 ; 1] * \text { DuDx; } \\
& \mathrm{y}=\mathrm{u}(1) * \mathrm{u}(2) ; \\
& \text { gamma }=0.01 ;
\end{aligned}
$$

beta $=0.01$;

$\mathrm{F}=\left(\operatorname{gamma} * \operatorname{beta}^{*} \operatorname{sqrt}(\mathrm{y})\right) /\left(1+\operatorname{gamma} * \operatorname{beta}^{*}(\operatorname{sqrt}(\mathrm{u}(2) /\right.$ $\mathrm{u}(1))))$;

$\mathrm{F} 1=(\operatorname{gamma} * \operatorname{sqrt}(\mathrm{y})) /($ beta $+\operatorname{gamma} *(\operatorname{sqrt}(\mathrm{u}(1) / \mathrm{u}(2))))$; $\%$ non linear terms

$\mathrm{s}=[-\mathrm{F} ;-\mathrm{F} 1]$;

$\%$ -

function $\mathrm{u} 0=\operatorname{pdex} 4 \mathrm{ic}(\mathrm{x}) ; \%$ create a initial conditions $\mathrm{u} 0=[1 ; 0.001]$

function $[\mathrm{pl}, \mathrm{ql}, \mathrm{pr}, \mathrm{qr}]=\mathrm{pdex} 4 \mathrm{bc}(\mathrm{xl}, \mathrm{ul}, \mathrm{xr}, \mathrm{ur}, \mathrm{t}) \% \mathrm{oreate}$ a boundary conditions

$$
\begin{aligned}
& \mathrm{pl}=[0 ; \mathrm{ul}(2)-1] ; \\
& \mathrm{ql}=[1 ; 0] ; \\
& \mathrm{pr}=[\mathrm{ur}(1)-1 ; 0] ; \\
& \mathrm{qr}=[0 ; 1] ;
\end{aligned}
$$

